\title{
Médiévales
}

Langues, Textes, Histoire

\section{Des religieux en quête de grâce : les suppliques adressées à la Pénitencerie apostolique par des clercs réguliers violents au $\mathrm{XV}^{\mathrm{e}}$ siècle}

Clerics Seeking Pardon: Petitions Brought before the Apostolic Penitentiary by Violent Regular Clerics in the Fifteenth Century

\section{Elisabeth Lusset}

\section{(2) OpenEdition}

\section{Journals}

Édition électronique

URL : http://journals.openedition.org/medievales/5472

DOI : 10.4000/medievales.5472

ISSN : 1777-5892

\section{Éditeur}

Presses universitaires de Vincennes

Édition imprimée

Date de publication : 20 décembre 2008

Pagination : 115-133

ISBN : 978-2-84292-221-4

ISSN : 0751-2708

\section{Référence électronique}

Elisabeth Lusset, « Des religieux en quête de grâce : les suppliques adressées à la Pénitencerie apostolique par des clercs réguliers violents au Xve siècle ", Médiévales [En ligne], 55 | automne 2008, mis en ligne le 20 mars 2011, consulté le 02 mai 2019. URL : http://journals.openedition.org/ medievales/5472 ; DOI : 10.4000/medievales.5472

Ce document a été généré automatiquement le 2 mai 2019.

Tous droits réservés 


\title{
Des religieux en quête de grâce : les suppliques adressées à la Pénitencerie apostolique par des clercs réguliers violents au $\mathrm{XV}^{e}$ siècle
}

\author{
Clerics Seeking Pardon: Petitions Brought before the Apostolic Penitentiary by \\ Violent Regular Clerics in the Fifteenth Century
}

\section{Elisabeth Lusset}

En avril 1465, les moniales bénédictines de Valleverde, dans le diocèse de Plaisance, adressent une supplique au pape afin de demander l'absolution pour le meurtre de leur abbesse. Elles expliquent qu'après une tentative d'empoisonnement infructueuse, elles ont payé un laïc pour étouffer leur supérieure. Cette affaire, enregistrée dans les registres de suppliques de la Pénitencerie, office pontifical chargé des absolutions, dispenses et licences accordées aux laïcs et aux clercs par le pape, est remarquable à plusieurs égards. D'une part, elle implique des femmes recluses, dont la criminalité est très difficile à saisir dans les archives monastiques ou diocésaines, d'autre part, ces moniales s'attaquent à leur supérieure et réitèrent leur tentative d'homicide ${ }^{1}$. Les archives de la Pénitencerie, longtemps fermées aux chercheurs car considérées comme relevant du secret de la confession, n'ont été rendues accessibles qu'en 1983, à certaines conditions de prudence, lorsqu'il apparut que la documentation de la période antérieure à 1564 était, sauf exception, relative aux cas de for externe ${ }^{2}$. Emil Göller avait initié les recherches sur la Pénitencerie en publiant, en 1913, une étude sur l'organisation administrative de cet office sans avoir eu, pour autant, accès aux registres de suppliques de la Pénitencerie ${ }^{3}$. À partir de 1983, Ludwig Schmugge et plusieurs autres historiens ont étudié, à travers les suppliques, les raisons juridiques poussant de nombreux fidèles à se rendre à Rome pour obtenir des rémissions à leurs infractions au droit canonique. L'importance des laïcs parmi les suppliants a été soulignée grâce à l'étude des dispenses et licences en matière 
matrimoniale. L'étude des suppliques a également permis, notamment grâce à Kirsi Salonen, d'éclairer les relations entre la papauté et les églises locales au $\mathrm{Xv}^{\mathrm{e}}$ siècle $^{4}$.

L'organe de la Pénitencerie a vu le jour dès le dernier tiers du XII ${ }^{e}$ siècle, mais seuls les registres de la seconde moitié $d u \mathrm{Xv}^{\mathrm{e}}$ siècle ont été conservés pour la période médiévale. Le fonds, exceptionnel par sa taille et son contenu, apparaît comme un réceptacle privilégié des affaires criminelles internes au monde conventuel ${ }^{5}$. Les religieux s'adressent à la Pénitencerie pour de multiples transgressions telles que l'apostasie, la simonie ou le vol. Une part importante des suppliques concerne les cas de violence exercés par des religieux à l'encontre d'un confrère ou un clerc. En vertu du canon Si quis suadente, ces religieux encourent une peine d'excommunication qui ne peut être levée que par le pape ${ }^{6}$. L'étude a été menée à partir du dépouillement des suppliques classées sous les titres de diversis formis, produites sous les pontificats de Pie II (1458-1464) et Paul II $(1464-1471)^{7}$. On recense, pour cette période, 245 suppliques adressées par des religieux ayant perpétré des actes de violence et des homicides à l'encontre de clercs réguliers ou séculiers ${ }^{8}$. Les cas d'apostasie ont été pris en compte lorsqu'un crime violent est à l'origine de la fuite du frère. Les religieux s'adressent au pénitencier majeur afin d'être absous des peines prononcées par leur supérieur, leur ordre ou leur évêque, ainsi que des sentences d'excommunication encourues ipso facto pour violence envers une personne ecclésiastique. Ils demandent également, lorsqu'ils sont clercs, à être dispensés de l'irrégularité contractée en commettant ce crime, qui les empêche d'exercer leurs fonctions sacerdotales ou d'être promus dans les ordres ${ }^{9}$. La supplique est adressée au pape avec l'indication du nom de baptême, du nom de famille, du grade du religieux, du nom du monastère et du diocèse d'origine. Le suppliant explique ce qu'il a fait, reconnaît sa culpabilité et annonce quel type de grâce il demande. Il ne rédige pas lui-même la requête mais recourt à un procureur rompu aux règles du droit canonique. Les registres ne contiennent pas le texte original des suppliques mais des copies abrégées, dans lesquelles sont consignées les informations nécessaires du point de vue juridique. À la fin de l'entrée, le copiste mentionne la décision de la Pénitencerie avec le nom et le titre de la personne qui a pris la décision (pénitencier majeur, régent). Les formules d'approbation varient selon le type de concession. Ces suppliques ne fournissent donc pas un récit brut des crimes commis. Dans la narration de l'épisode criminel, le suppliant cherche à la fois à présenter les faits avec exactitude et à minimiser sa culpabilité afin d'obtenir la grâce. On cerne donc moins la réalité de la violence que sa perception et sa mise en forme par les institutions pontificales ${ }^{10}$.

3 L'analyse de ces suppliques invite à s'interroger sur les raisons qui poussent les religieux coupables de violence ou d'homicide à recourir à la Pénitencerie. En effet, l'étude de la législation canonique en matière de violence commise par des religieux à l'encontre des personnes ecclésiastiques montre que, dans la seconde moitié $d u \mathrm{xv}^{\mathrm{e}}$ siècle, les religieux ne sont plus astreints à recourir à Rome pour être absous de la sentence d'excommunication. La papauté a, en effet, progressivement délégué ce pouvoir aux supérieurs des maisons religieuses, tout en gardant un droit de réserve sur les excès "difficiles et énormes». Cependant de nombreux religieux continuent de recourir à l'office pontifical, y compris pour des cas de criminalité « ordinaire ». Quel rôle joue alors la Pénitencerie dans la correction et la grâce de ces criminels réguliers venus de toute l'Europe ? À quels besoins répond-t-elle d'un point de vue procédural ? Il s'agit, en définitive, de mieux saisir le fonctionnement de la justice interne aux communautés conventuelles et les stratégies déployées par les religieux pour obtenir la grâce. L'analyse 
sera tout d'abord consacrée à l'évolution de la législation canonique en matière de violence commise par des religieux à l'encontre de leurs confrères. Ces dispositions normatives seront ensuite confrontées à l'étude des cas effectivement soumis à la connaissance de la Pénitencerie dans les années 1460-1470. Enfin, à travers l'étude de quelques figures-type de suppliants coupables de violence ou d'homicide, nous tenterons de mieux comprendre les raisons du recours à Rome.

Depuis la promulgation du canon Si quis suadente au concile de Latran II par Innocent II en 1139 , toute atteinte physique à la personne d'un clerc ou d'un religieux est punie d'une peine d'excommunication latae sententie. L'excommunication découle ipso facto de l'accomplissement de l'acte visé par le canon qui l'interdit. Le pape se réserve l'absolution de ces cas de violence. Aucun évêque ne peut absoudre cette sentence d'excommunication avant d'avoir reçu mandat du pape, devant lequel le coupable est tenu de se présenter pour être jugé ${ }^{11}$. Par la suite, les papes Alexandre III (1159-1181), Clément III (1187-1191) et Célestin III (1191-1198) réduisent la portée du canon, en formulant des exceptions à son application. Les religieux ayant commis une voie de fait en état de légitime défense échappent à l'excommunication. De même, les jeunes religieux ne voient pas leurs actes de violence sanctionnés, quia eos aetas excusat ${ }^{12}$. Par ailleurs, Alexandre III autorise les moines et les chanoines réguliers ayant frappé un membre de leur communauté à être corrigés et absous par leur abbé13. En 1202, Innocent III étend cette exception aux moniales dans la décrétale De monialibus. Si une religieuse frappe une autre religieuse, un convers ou un clerc séculier, elle sera absoute par l'évêque ${ }^{14}$. Ces décisions sont reprises dans la collection de droit canonique du Liber extra en 1234. La papauté continue cependant de se réserver la compétence de certains cas de violence. Dans une décrétale adressée au maître et aux frères de l'Hôpital de Saint-Jean-de-Jérusalem, Honorius III (1216-1227) prescrit que les moines et chanoines réguliers qui se seront frappés mutuellement ne seront pas envoyés au Siège apostolique mais soumis à la discipline de l'abbé. Si la discrétion de l'abbé ne suffit pas, l'évêque doit lui venir en aide. Cependant si l'excès est « difficile et énorme » (excessus difficilis et enormis), le religieux criminel doit se rendre à la curie ${ }^{15}$. Les textes ne précisent cependant pas quels cas de violence ressortissent à la catégorie d'« excès difficile et énorme ». Julien Théry a récemment montré qu'à partir du XII ${ }^{\mathrm{e}}$ siècle, dans les lettres pontificales, «l'enormitas n'[est] plus synonyme de simple " excès »; elle désign[e] désormais ce qui [est] à la fois contraire aux règles canoniques et extrêmement grave, avec une forte connotation d'immoralité ». L'expression "excès énormes » désigne une infraction majeure, souvent des faits de simonie, d'homicide ou d'incontinence et qualifie un crime démesuré mettant en cause la toute puissance de l'Église ${ }^{16}$. Dans le cas de la décrétale d'Honorius III, on pourrait émettre l'hypothèse que l'expression désigne, non plus de simples violences entre confrères, mais des agressions envers des prélats ou des violences provoquant une effusion de sang ou la mutilation d'un membre. Dans tous les cas, l'absence de définition stricte des cas ressortissant à cette catégorie permet à la papauté de se réserver un large spectre d'affaires.

$5 \quad$ Les dispositions du droit général de l'Église sont relayées par les dispositions particulières prises par les papes à l'égard des ordres exempts. Prenons ainsi l'exemple de l'ordre de Cîteaux, qui se caractérise par son importante production législative. Dans la seconde moitié du XII ${ }^{\mathrm{e}}$ siècle, Alexandre III confère aux abbés le privilège d'absoudre les moines de l'excommunication encourue pour avoir commis un incendie ou frappé un clerc ou un religieux ${ }^{17}$. Par ce privilège, les moines cisterciens n'ont plus besoin de recourir à Rome. 
Cependant la délégation de la potestas absolvendi n'est pas entière. Seuls les cas de violence entre moines sont concernés. En 1234, Grégoire IX confirme le privilège d'absoudre les moines et les convers cisterciens de toute excommunication encourue, précise-t-il, en cas de violence commis entre eux (in seipsos) ${ }^{18}$. La codification cistercienne de 1237 opère une première synthèse en stipulant que si un moine ou un convers frappe un autre religieux, il doit être excommunié immédiatement jusqu'à ce que l'abbé ou le prieur l'absolve. En revanche, si ce même moine ou convers frappe un abbé, il ne peut être absous que par le pape. Les voies de faits sur un supérieur font partie des cas réservés par la papauté ${ }^{19}$. Par ailleurs, l'ordre cistercien reste tenu de porter les cas graves à la connaissance du pape. En 1255, dans une bulle qui ne concerne pas spécifiquement les cas de violence, Alexandre IV concède aux abbés cisterciens la faculté de lever les sentences d'excommunication et de dispenser les moines de toute irrégularité, sauf s'il s'agit d'un "excès très grave et énorme ». Dans ce cas, ils doivent être adressés au Siège apostolique ${ }^{20}$. Le chapitre général de 1255 reprend le texte. Les abbés peuvent absoudre et dispenser leurs sujets dans les cas où ceux-ci ont encouru l'excommunication ou l'irrégularité, pourvu que ces cas ne soient pas tels qu'à cause de leur "énormité », il faille recourir au pape ${ }^{21}$. L'ensemble de cette législation est synthétisée dans le Libellus diffinitionum de 1257. Lorsque les définiteurs exposent le privilège d'Alexandre IV, ils rappellent que la faculté d'absolution et de dispense doit être utilisée avec discernement. Comme il est difficile de savoir à qui, du Siège apostolique ou du chapitre général, le cas est réservé, tout cas douteux doit être discuté lors du chapitre ${ }^{22}$. Dans une autre distinction, les définiteurs reprennent mot pour mot la codification de 1237. Si un religieux cistercien frappe un abbé, il ne peut être absous que par le pape. Ces dispositions ne seront pas modifiées dans les recueils de codification ultérieurs ${ }^{23}$. D'après la législation cistercienne, le pape conserve donc un droit de réserve pour les « excès difficiles et énormes » sans que, pour autant, le contenu de cette catégorie soit précisé. Par ailleurs, les cas de violence à l'encontre d'un supérieur ne peuvent être absous que par le pape.

Cet examen du droit canonique et du droit particulier des ordres religieux permet de voir que tous les cas de violence intra-monastiques ne relèvent pas de la compétence pontificale, du fait des dérogations accordées à l'application du canon Si quis suadente. La violence commise entre confrères peut être absoute par les supérieurs religieux. En revanche, l'absolution de l'excommunication encourue pour agression d'un supérieur et pour des cas «énormes et difficiles» reste du ressort de la curie pontificale. Il s'agit à présent de confronter ces dispositions normatives aux cas rencontrés dans les archives des chapitres généraux et de la Pénitencerie afin de mieux cerner à quelle juridiction revient la correction des religieux criminels.

7 Les registres des chapitres généraux clunisien et cistercien mentionnent des cas de moines impliqués dans des crimes dont l'absolution est réservée au pape. En 1357, Hugo de Tuella, moine clunisien de Bourbon-Lancy (diocèse d'Autun) frappe un confrère, Thomas. Les définiteurs décident que le moine sera cité à comparaître devant le prieur claustral de Cluny afin de recevoir la discipline régulière pour ses excès et qu'il sera transféré dans un autre monastère. Mais "parce que l'excès de cette agression (verberationis) apparait difficile et énorme, Hugo doit être absous par le Siège apostolique ${ }^{24}$ ». Le chapitre général clunisien se conforme donc aux décisions pontificales et notamment à la décrétale d'Honorius III reprise dans le Liber extra en $1234^{25}$. En 1433 , Gumo Andinol, moine clunisien de Contamine (diocèse de Genève) frappe un clerc au visage et le blesse à l'œil. Le clerc perd l'usage de la vue pendant quatre jours. Gumo 
célèbre la messe dans l'église contre l'avis du sous prieur. Les définiteurs décident que le moine doit recourir au pape afin d'obtenir l'absolution et la dispense pour l'excommunication et l'irrégularité contractées ${ }^{26}$. De même, en 1467, le chapitre général cistercien ordonne à Jean de Steffelstein, moine de Schönau (diocèse de Worms), qui a frappé l'abbé de Morimond, commissaire de l'ordre, de s'en remettre au pape pour l'absolution de son crime ${ }^{27}$. Dans les trois exemples cités, le chapitre se déclare incompétent pour absoudre et décide de recourir à l'autorité suprême de l'Eglise. Ces crimes se caractérisent par leur gravité et leur démesure, en ce qu'ils manifestent une violence exacerbée ou prennent pour cible des supérieurs.

8 Cependant, dans la plupart des cas de demande d'absolution et de dispense, les chapitres clunisien et cistercien se déclarent compétents. En 1440, Denis de Valle, moine cistercien de Saint-André de Gouffern (diocèse de Bayeux) frappe un profès, Gilles Bernier usque ad enormem sanguinis effusionem. Le chapitre déclare que ce cas lui est réservé : il absout le moine et l'autorise à accéder à tous les grades au sein du couvent, y compris à la charge abbatiale ${ }^{28}$. Malgré le sang versé, ce crime, commis entre pairs, reste du ressort de la justice interne à l'ordre. Certains cas attestent de l'importance de la potestas absolvendi que le chapitre général cistercien s'octroie. En 1441, un moine, Jean Le Picard agresse l'abbé de Lanvaux (diocèse de Vannes). Le chapitre dispense le moine et autorise son abbé à l'absoudre de la sentence d'excommunication en précisant que l'acte a été perpétré absque maxima violentia ${ }^{29}$. Les sources judiciaires internes aux ordres corroborent les observations faites concernant la législation canonique. Les chapitres généraux ont une large compétence en matière de violence perpétrée au sein des maisons religieuses. Certains cas restent cependant du ressort du pape, notamment les cas de violence grave $d u$ fait des blessures engendrées ou du fait du statut de la victime. Il s'agit donc de savoir si ces cas réservés sont, en particulier, traités par l'office de la Pénitencerie, dont on a vu qu'il enregistrait un certain nombre de suppliques concernant des religieux violents.

Un examen approfondi des suppliques de la Pénitencerie infirme ces hypothèses. Il n'y a pas, semble-t-il, de correspondance entre les cas réservés au pape dans la législation et les cas enregistrés par la Pénitencerie dans les suppliques de diversis formis. En effet, ce ne sont pas les suppliants coupables d'atteintes à la personne d'un supérieur ou de crimes «difficiles et énormes » que l'on voit apparaître dans les archives de cet office. Dans les cas de crimes violents, l'effusion de sang n'est ainsi mentionnée que dans $18 \%$ des suppliques étudiées. Dans près de $20 \%$ des cas, les suppliants précisent qu'il a eu coup mais sans effusion de sang. Si l'on observe ensuite le profil des victimes, on constate que les supérieurs des maisons religieuses ne sont victimes que dans $16 \%$ des cas. Les suppliants sont avant tout des religieux s'attaquant aux membres du clergé séculier, cible des attaques dans $28 \%$ des cas et surtout à leurs confrères. Les moines, chanoines et frères représentent $46 \%$ des victimes. Lorsque l'on s'intéresse aux liens entre les coupables et les victimes, on constate que, dans plus de la moitié des cas, des moines, chanoines, frères s'attaquent à leur socius. Plus de $55 \%$ des moines s'attaquent à d'autres moines, $64 \%$ des chanoines réguliers à d'autres chanoines et $55 \%$ des frères mendiants s'en prennent à leurs confrères. La Pénitencerie enregistre également des cas d'homicides commis par des religieux. Contrairement à l'iniectio manuum, l'absolution d'un homicide n'est pas réservée au pape. Il incombe aux juges ecclésiastiques locaux de décider si un cas mérite ou non de rester sous leur juridiction ordinaire. Cependant lorsque l'agresseur ou la victime apparaissent aux ordres majeurs, le recours à Rome est encouragé ${ }^{30}$. Dans les registres étudiés, seules $10 \%$ des suppliques relatives à une voie de fait concernent des 
cas d'homicide entre religieux. Les supérieurs ne sont victimes que dans $15 \%$ des cas. La Pénitencerie ne traite donc pas en particulier les cas de violences et d'homicides à l'encontre des supérieurs.

10 Par ailleurs, ces agressions ne sont jamais qualifiées, dans les suppliques, de crimes «difficiles et énormes». Les suppliants s'attachent à démontrer que leur crime n'appartient pas à cette catégorie. Le 25 avril 1466, Nicolas Dauxe, moine prêtre clunisien d'Abbeville (diocèse d'Amiens) explique qu'il a frappé son prieur, Antoine Mustel, dans l'église mais sans provoquer d'effusion de sang ni commettre d'autre excès « difficile et énorme " (citra tamen sanguinis effusione in ecclesia eiusdem monasterii iniecit absque tamen alio excessu difficili vel enormi) ${ }^{31}$. Cette clause participe de la stratégie défensive adoptée par certains suppliants pour obtenir la grâce. Les religieux, avec l'aide des procureurs qui rédigent la supplique, informent le récit en fonction de la législation. Il s'agit de prouver que le crime commis est moins grave qu'on pourrait le penser, qu'il rentre dans les exceptions définies par le droit canonique et que, par conséquent, le coupable peut être gracié. Ainsi l'arme du crime est souvent décrite comme un petit couteau servant à couper le pain, afin d'éviter que le religieux ajoute à son crime le délit de port d'arme, proscrit par son état. Le suppliant dit avoir agi pour se défendre (vim vi repellendo et se defendendo) ou avoir frappé par inadvertance. La blessure est présentée comme "modique $»^{32}$. Lorsque le suppliant est l'agresseur, il ne minore pas l'importance des blessures infligées mais explique qu'il s'est trompé de victime. En 1464, Philippe Tuonmelni, moine prêtre bénédictin de Saint-Sauveur-de-Redon (diocèse de Rennes), déclare que « croyant frapper un laïc avec une épée, il a frappé par hasard un religieux de l'ordre jusqu'à l'effusion de sang ». Il explique que la victime s'est pleinement rétablie et demande à être absous de l'excommunication et de ses péchés ${ }^{33}$. Le récit du crime vise moins à retranscrire les étapes de l'agression qu'à multiplier les circonstances atténuantes qui diluent la responsabilité pénale du suppliant ${ }^{34}$. La déformation de la réalité ne peut cependant excéder certaines limites. La lettre pontificale n'est délivrée qu'après vérification des informations données par les autorités locales. Ainsi en 1450, Johannes Limborgh, prévôt de l'abbaye bénédictine de Werden (diocèse de Cologne), frappe son familier avec une épée et le blesse à la main gauche, d'une blessure "non mortelle » précise la supplique. Quelques jours plus tard, la victime meurt. Le régent de la Pénitencerie accorde la grâce à condition qu'un médecin ou qu'un expert assure que la blessure n'était pas mortelle (si iudicio medicorum seu peritorum vulnus letale non fuit) ${ }^{35}$.

11 Une affaire criminelle, enregistrée dans les archives du chapitre général cistercien et dans celles de la Pénitencerie, permet de mieux comprendre les stratégies déployées par les suppliants. En 1461, les définiteurs cisterciens envoient plusieurs abbés enquêter sur la participation de Luc Dessossuys, moine de la Trappe (diocèse de Sées), au meurtre de l'abbé de Tironneau, un monastère voisin (diocèse du Mans). L'abbé a été aveuglé et son cerveau transpercé ${ }^{36}$. Le crime est décrit de façon détaillée par les définiteurs habituellement très laconiques. Ils soulignent le caractère sacrilège du meurtre. La gravité du crime est confirmée par l'importance de la délégation envoyée pour enquêter sur le crime ${ }^{37}$. Cependant les définiteurs n'évoquent à aucun moment la nécessité de recourir à Rome pour régler l'affaire ${ }^{38}$. L'année suivante, le 20 février 1462, la Pénitencerie enregistre la supplique de Jean Rufi, moine de Tironneau. Il déclare avoir tué son abbé avec d'autres moines (diabolo instigante una cum quibusdam sociis suis monachis dicti monasterii abbatem suum interfecit) et avoir fui pour se rendre à la Curie romaine. Il s'agit probablement d'un des complices de Lucas Dessosuys, qui décide de contourner le 
chapitre général pour obtenir directement l'absolution du Saint Siège ${ }^{39}$. La supplique de Jean Rufi provoque l'intervention de l'évêque du Mans, chargé de vérifier l'exactitude des faits. Le suppliant est absous de la sentence d'excommunication et du crime d'abbaticide ( abbaticidii reatum) mais ne peut plus exercer dans les ordres sacrés. La comparaison des deux comptes-rendus du crime, dans les archives du chapitre général et dans la supplique, permet de constater que le suppliant se garde d'évoquer la manière sacrilège dont le meurtre a été commis. On pourrait interpréter le silence de Jean Rufi comme une tentative pour minorer l'importance de son crime afin d'obtenir plus facilement la grâce. Il ne faut cependant pas se laisser abuser par la formulation laconique de l'enregistrement. La gravité du crime n'échappe pas à la Pénitencerie. Jean Rufi reste suspendu et ne reçoit pas une absolution pleine et entière, contrairement à la plupart des suppliants, qui obtiennent ce qu'ils demandent ${ }^{40}$. L'analyse des suppliques se doit donc d'être attentive à toutes les clauses mentionnées. Il convient enfin de souligner qu'il s'agit d'un des très rares cas d'abbaticides recensés entre 1458 et 1474 .

Au terme de cette analyse, on peut avancer que la Pénitencerie semble destinée à traiter des cas de criminalité « ordinaire » et non des agressions à l'égard des supérieurs ou des cas "difficiles et énormes". Dans la plupart des suppliques de diversis formis, la qualification du crime ne donne pas lieu à une narration détaillée ${ }^{41}$. Les formules d'enregistrement sont très succinctes, car il s'agit simplement de garder la mémoire des lettres de grâce concédées par la Pénitencerie. La plupart des suppliants sont des religieux qui ont frappé un confrère dans un accès de colère ou de rage, souvent provoqué par des paroles offensantes. Le 9 mai 1465, Jean Vérard, moine profès bénédictin de SaintMartin de Sées, explique qu'il a frappé au visage un des frères après que celui-ci l'a injurié ${ }^{42}$. Il semblerait que les cas hors norme, les cas réservés par le pape, pour lesquels les ordres religieux n'ont pas la compétence de dispenser et d'absoudre soient traités par d'autres organes de la Curie, notamment la Chancellerie ${ }^{43}$. Les religieux peuvent également recourir aux légats pontificaux. En 1472, le chapitre général cistercien enregistre l'absolution, par un légat du pape, de Jean Walgemunt, moine d'Aldersbach (diocèse de Passau), du crime d'homicide commis avant son entrée dans les ordres ${ }^{44}$. Le constat selon lequel les suppliques exposent des cas de criminalité ordinaire pose cependant problème. La Pénitencerie présente le paradoxe de conférer des grâces à des religieux qui peuvent déjà les obtenir au sein de leur ordre car ceux-ci ont la compétence pour délivrer des dispenses et des absolutions. Comment expliquer alors que les moines recourent à une procédure coûteuse en temps et en argent alors qu'ils pourraient être absous par les instances judiciaires internes à leur ordre ${ }^{45}$ ? À quels « besoins judiciaires » la Pénitencerie répond-t-elle? L'analyse des suppliques de diversis formis conduit à s'interroger sur le parcours judicaire des religieux délinquants, les recours qui s'offrent à eux et les stratégies qu'ils déploient face à la justice régulière.

Le recours à la Pénitencerie pour absoudre des cas de violence relevant, en théorie, des supérieurs ou des chapitres généraux invite tout d'abord à s'interroger sur le profil des suppliants. L'exemption joue-t-elle un rôle dans le recours privilégié ou non à cet office pontifical? Les registres de diversis formis comptent peu de suppliants cisterciens et clunisiens. Pour la période étudiée, ils représentent $6,7 \%$ et $4,7 \%$ des religieux violents. Les ordres mendiants sont mieux représentés avec, entre autres, 8,3\% de Franciscains. Cependant la majorité des suppliants réguliers sont des chanoines réguliers $(17 \%)^{46}$ et surtout des Bénédictins $(41,9 \%)^{47}$. Au xv $v^{\mathrm{e}}$ siècle, la plupart des religieux ont intégré une structure d'ordre, avec notamment la tenue d'un chapitre général ou provincial et 
l'exercice de la visite canonique. Cependant, tous ne se caractérisent pas par le même degré de cohésion et de centralisation. Les Cisterciens, les Clunisiens, les ordres mendiants et l'ordre de Prémontré présentent une véritable organisation d'ordre ${ }^{48}$. En revanche, la papauté ne parvient pas à imposer ces structures administratives aux chanoines réguliers indépendants et aux moines bénédictins ${ }^{49}$. La rareté des suppliants cisterciens et clunisiens laisse supposer qu'un grand nombre d'absolutions et de dispenses pour des sentences d'excommunication pour des cas de violence est accordé au sein de l'ordre, conformément à la législation précédemment étudiée. Si l'on examine les registres du chapitre général cistercien entre 1458 et 1474, on décompte 6 cas de dispenses et d'absolution pour 15 crimes violents enregistrés ${ }^{50}$. Les instances administratives et judiciaires centralisées des ordres exempts, anciens et nouveaux, prennent donc en charge la correction aussi bien que la grâce de leurs criminels. La forte proportion de moines bénédictins s'explique, quant à elle, par leur importance numérique mais peut aussi être motivée par l'absence d'une structure d'ordre et d'un chapitre général susceptible d'absorber une partie de la potestas absolvendi pontificale. Ces moines dépendent soit de l'évêque local, soit du pape lorsqu'ils sont directement soumis à lui par un privilège. En août 1472, Jean Hansgor, moine prêtre bénédictin de Mehrerau (diocèse de Constance) sedi apostolice nullo medio subiecti, demande à être absous et dispensé pour avoir menacé avec une arme un prêtre et lui avoir extorqué de l'argent ${ }^{51}$. On peut supposer que ces monastères directement sujets du pape recourent plus facilement à la Pénitencerie. Cependant, dans le cas des Bénédictins comme des autres frères, la Pénitencerie n'offre pas le chemin le plus direct vers l'absolution. C'est donc qu'elle présente, aux yeux des religieux, des avantages que n'offrent pas les évêques, les supérieurs locaux ou les chapitres généraux.

14 En premier lieu, les religieux violents n'obtiennent pas toujours gain de cause auprès des instances judiciaires de leur ordre. En 1442, François Guyse, moine cistercien de Foigny (diocèse de Laon) frappe un autre religieux dans l'église du monastère, sans provoquer d'effusion de sang. Le prieur lui interdit de pénétrer dans l'église. Le moine s'adresse au sous prieur, mais celui-ci refuse de l'absoudre, expliquant que le moine ne peut s'adresser qu'à l'abbé. Le frère recourt alors à un autre confesseur, et une fois confessé, célèbre à nouveau l'office. Il est puni d'une peine de prison puis absous par l'abbé sur ordre du chapitre général ${ }^{52}$. François Guyse, bien qu'ayant la possibilité d'être absous par son abbé, semble s'y refuser. Des contentieux avec le supérieur peuvent en effet conduire les religieux à rechercher leur salut auprès d'autres instances. Le 10 avril 1475, Ermelina de Osselburgen, moniale cistercienne d'olsperg (diocèse de Bâle) explique qu'elle a été accusée de divers délits par ses ennemis, en présence du supérieur et de l'abbé visiteur. La moniale tente de prouver son innocence, mais l'abbé l'enferme en prison. Ermelina y reste six mois avant d'être délivrée par des consœurs et de se réfugier chez ses parents ${ }^{53}$. Face à une justice présentée comme expéditive, la moniale apostate choisit de recourir, non pas au chapitre général, qui, en la personne de l'abbé-visiteur, a montré sa défaillance, mais à l'instance judiciaire suprême, la justice du pape.

15 Il semblerait, à l'instar de cette moniale apostate, que la Pénitencerie soit le recours privilégié des religieux qui, une fois leur crime perpétré, ont fui leur couvent. En 1455, Marc Kamrer, moine bénédictin de Saint-Pierre de Thierhaubten (diocèse d'Augsbourg) explique qu'il a fui après avoir blessé mortellement son abbé. Il vagabonde pendant six mois puis se rend à Rome où il reçoit l'absolution et l'autorisation de revenir dans son couvent $^{54}$. La gravité du crime, une blessure mortelle infligée à un supérieur, laisse 
présager un dur châtiment auquel le moine tente d'échapper. Les religieux fuient également pour abréger la durée de leur peine. Le 19 janvier 1461, Paul Borchard, moine prêtre cistercien de Dargun (diocèse de Kammin) frappe un prêtre avec un couteau. Il est emprisonné par son supérieur pendant sept semaines mais parvient à s'enfuir. Il vagabonde, entend des confessions et administre les sacrements. Il écrit au pape pour demander à être absous, dispensé et transféré dans une autre maison ${ }^{55}$. Cependant, en cherchant à éviter la correction par la fuite et en refusant la peine imposée par leur supérieur, les religieux aggravent le crime initialement commis ${ }^{56}$. Bien que la règle de saint Benoît et les codifications ultérieures, comme la bulle Pastor bonus de Benoît XII en 1335, appellent à la clémence envers les fugitifs repentants (le fugitif peut être reçu jusqu'à trois fois), les supérieurs sont souvent réticents à l'idée d'accueillir un criminel doublé d'un apostat ${ }^{57}$. Les lettres délivrées par l'office pontifical fournissent aux religieux pénitents un argument de poids pour réintégrer leur couvent. Le recours à la Pénitencerie est donc le fait de religieux criminels et désobéissants, qui refusent de se soumettre à la correction de la justice régulière. Les suppliques offrent ainsi quelques figures de ces moines en rupture de ban. Le 15 juillet 1470, Jean de Piron, chanoine augustin sous-diacre de Saint-Barthélemy de Noyon, raconte qu'il a sorti un petit couteau et frappé plusieurs fois à la tête avec ses poings un frère, Thomas Soliart, en raison de paroles injurieuses. L'abbé l'enferme pendant quatre jours. Le chanoine parvient à s'échapper de nuit et attaque à nouveau Thomas, après les matines, alors que celui-ci quitte le dortoir. Jean porte de multiples coups à la tête de Thomas avec un bâton, lui casse le bras, sans faire jaillir le sang, puis s'enfuit. Ses exactions ne cessent pas pour autant. Il s'attaque violemment à plusieurs reprises à des clercs séculiers et réguliers. Dans la supplique, Jean demande à être absous de l'excommunication et du crime d'apostasie. Il souhaite retourner dans son monastère, ses victimes ayant guéri sans être inhabiles ${ }^{58}$. En s'attaquant par deux fois à la même personne et en continuant ses violences en dehors du cloître, Jean s'apparente à un chanoine incorrigible. Il persévère dans le péché et semble avoir atteint un point de non-retour qui le rend indigne du pardon. La justice monastique réserve de lourdes peines (prison, transfert, dégradation) à ce type de délinquants. Dans les cas les plus graves, le religieux peut être expulsé de l'ordre ${ }^{59}$. Seule la potestas absolvendi du pape semble alors assez puissante pour assurer à ces religieux la réintégration dans la communauté.

L'obtention d'une lettre de la Pénitencerie ne garantit pourtant pas toujours au suppliant qu'il verra ses volontés exaucées. Le 29 février 1456, Jean Waltum, moine bénédictin de Saint-Symphorien à Metz, explique qu'il a apostasié pendant plusieurs années et célébré des messes. Il a été absous et dispensé par le pape mais l'abbé refuse de le recevoir. Le moine vagabonde pendant un an, retourne voir l'abbé et le supplie de le recevoir ou de le transférer, l'abbé refuse à nouveau. L'apostat rédige alors une fausse autorisation en utilisant le sceau de l'abbé et entre dans le monastère de Saint-Pierre de Molesme (diocèse de Langres). Il se rend ensuite à Rome pour régulariser sa situation ${ }^{60}$. L'autorité pontificale ne suffit donc pas toujours à emporter l'accord des instances locales. Les responsables des ordres, conscients de la facilité avec laquelle les religieux obtiennent ces absolutions, rechignent à obéir aux décisions extérieures. Dès 1278, le chapitre général cistercien déclare que toute lettre de la Pénitencerie, autorisant un fugitif à desservir l'autel à son retour, serait considérée comme nulle et non avenue ${ }^{61}$. Les suppliques dressent le portrait de suppliants fin stratèges qui savent défendre au mieux leurs intérêts et utiliser les recours judiciaires qui s'offrent à eux. 
17 En définitive, les suppliques de diversis formis des années 1458-1471, adressées par des religieux impliqués dans un crime violent, ne ressortissent pas à la catégorie des cas réservés au pape. Au contraire, la Pénitencerie se charge d'absoudre et de dispenser des délits « ordinaires » tels que les coups échangés entre confrères au cours d'une dispute. Dès la fin $\mathrm{du} \mathrm{XII}^{\mathrm{e}}$ siècle, les ordres religieux obtiennent la faculté d'absoudre de la sentence d'excommunication encourue ipso facto pour ce type de cas. Les religieux continuent cependant $\mathrm{au} \mathrm{XV}^{\mathrm{e}}$ siècle à recourir à Rome pour être graciés. Le Siège apostolique apparaît aux suppliants comme la seule autorité capable de dispenser une grâce sans limites. Les lettres d'absolution concédées par la Pénitencerie constituent en effet des pièces décisives susceptibles d'influer sur les décisions des juridictions locales qui, si elles sont compétentes en la matière, se montrent parfois peu clémentes avec les religieux délinquants. L'attitude des réguliers face à la justice ecclésiastique n'est pas sans rappeler celle des sujets du roi de France dans le cadre de la justice royale retenue à la fin du Moyen Âge. L'étude des sources de la Pénitencerie s'inscrit de ce fait dans la perspective plus large d'une histoire de la procédure criminelle au sein des justices laïques et ecclésiastiques. Les suppliques invitent à s'interroger sur l'utilisation par les justiciables d'un système juridictionnel certes hiérarchisé mais souple dans son usage. Le recours à la Pénitencerie ne relève cependant pas seulement d'une utilisation calculatrice des institutions ecclésiastiques. Les suppliques attestent, à l'instar de ce qui a été observé pour les laïcs, du poids de la législation canonique. Elles apportent un éclairage nouveau sur l'idée du déclin de l'idéal régulier à la fin du Moyen Âge. Si les suppliques exposent les désordres internes à la vie conventuelle, où règnent une discorde et une violence aux antipodes des idéaux de paix et de charité, elles montrent aussi des religieux sur la voie de la résipiscence. Ces moines, chanoines et frères, qui ont transgressé le droit canonique, s'engagent dans une procédure coûteuse en temps et en argent afin de réintégrer leur communauté, lavés de la macule de l'irrégularité et de l'infamie ${ }^{62}$.

\section{NOTES}

1.Petunt se ab excommunicationis sententia et homicidii reatu absolvi. L'absolution consiste ici à libérer les moniales de la sanction canonique d'excommunication mais également à remettre le péché d'homicide. Archivio Segreto Vaticano (désormais abrégé en : Asv), Penitenzieria apostolica Reg. Matrim. et Div. 12, fo 67r, éd. dans F. TAMBURINI, Santi e peccatori, Confessioni e suppliche dai Registri della Penitenzieria dell'Archivio Segreto Vaticano (1451-1586), Milan, 1995, cas $n^{\circ} 17$.

2.Le fait que la Pénitencerie apostolique ait refusé de mettre ces sources à disposition du public pendant près de 70 ans, depuis la découverte d'E. Göller en 1913 jusqu'en 1983, est officiellement dû aux craintes qu'elles pussent contenir des éléments relevant du sceau du secret sacramentel (sigillum confessionis). En réalité, les registres originaux concernent des cas relatifs à la sphère judiciaire publique. Sur la question de la distinction entre for interne et externe à la Pénitencerie, voir A. FosSIER, « La pénitencerie pontificale en Avignon ( $\mathrm{XIV}^{\mathrm{e}}$ siècle) ou la justice des âmes comme style de gouvernement ", dans Les 
Justices d'Église dans le Midi ( $\mathrm{xI} \mathrm{e}^{\mathrm{e}} \mathrm{XV^{ \textrm {e } }}$ siècle), Cahiers de Fanjeaux, 42, Toulouse, 2007, p. 199-239, ici p. 208.

3.C'est seulement après avoir publié son ouvrage sur la Pénitencerie en 1913 que E. Göller apprend du cardinal pénitencier que les archives n'ont pas été totalement perdues lorsque Napoléon a transféré tous les documents du Vatican à Paris en 1809. Il vient cependant de quitter Rome pour devenir professeur de droit canonique à l'université de Freiburg et n'aura pas l'occasion de publier de travaux sur ces archives nouvellement découvertes. E. GÖLLER, Die päpstliche Pönitentiarie von ihrem Ursprung bis zu ihrer Umgestaltung unter Pius V, 4 vol. Rome, 1907-1911, E. GöLLER, « Das alte Archiv der päpstlichen Pönitentiarie », Römische Quartalschrift, Supplementband, 20, Rome-Fribourg, 1913, p. 1-19.

4.L. SCHMUGGE dir., Die Supplienregister der päpstlichen Pönitentiarie aus der Zeit Pius'II, Tübingen, 1996, P. ostinelli, Penitenzieria Apostolica. Le suppliche alla S.P.A. provenienti dalla diocesi di Como, 1438-1484, Milan, 2003, M. MAILLARD-LuYPAERT, Les Suppliques de la Pénitencerie apostolique pour les diocèses de Cambrai, Liège, Thérouranne et Tournai (1410-1411), Bruxelles-Rome, 2003, K. SALONEN et C. KRÖTZL dir., The Roman Curia, the Apostolic Penitentiary and the «Partes » in the Later Middle Ages, Rome, 2003, H. Millet dir., Suppliques et requêtes, le gouvernement par la grâce en Occident ( $\mathrm{xII}^{\mathrm{e}}-\mathrm{XV}^{\mathrm{e}}$ siècle), Rome, 2003, A. MEYER dir., Päpste, Pilger, Pönitentiarie, Festschrift für Ludwig Schmugge zum 65. Geburtstag, Tübingen, 2004, G. JARITZ dir., The Long Arm of Papal Authority : Late Medieval Christian Peripheries and Their Communication with the Holy See, Budapest, 2005, W. MÜLLER, «Violence et droit canonique : les enseignements de la Pénitencerie apostolique (XIII ${ }^{\mathrm{e}}-\mathrm{XVI}^{\mathrm{e}}$ siècle) », Revue historique, 644, 2007, p. 771-796. Les suppliques des diocèses germaniques ont été éditées dans le Repertorium Poenitentiariae Germanicum, Verzeichnis der in den Supplikenregistern der Pönitentiarie vorkommenden Personen, Kirchen und Orte des Deutschen Reiches, L. ScHMUGGE éd., 7 vol., Tübingen, 1996-2005 (désormais abrégé en : RPG). L'édition couvre les pontificats d'Eugène IV à Sixte IV, de 1431 à 1484 .

5.On conserve un registre datant de 1409 et de 1438-1439, puis à partir de 1449, un registre par année, avec quelques lacunes. 746 volumes de suppliques ont été conservés pour la période allant du XV siècle à 1890 . K. SALONEN, The Penitentiary as Well of Grace in the late Middle Ages. The Example of the Province of Uppsala 1448-1527, Helsinki, 2001. Ces sources permettent d'avoir connaissance d'affaires internes aux maisons religieuses, non mentionnées dans les sources des échelons inférieurs comme les documents de visite. 6.Concile de Latran II, c. 15 (1139), repris dans Décret de Gratien, C. 17.4.29, Corpus juris canonici, E. FRIEDBERG éd., Leipzig, 1881, réimp. Clark (New Jersey), 2000, t. I, col. 822 (désormais abrégé en : FRIEDBERG).

7.Asv, Penitenziera Ap., Reg. Matrim et Div. 7 à 19. L'enregistrement des suppliques ne devient systématique qu'à partir de Pie II. Les cahiers dans lesquels sont transcrites les copies de suppliques sont reliés au sein d'un volume contenant en théorie tous les types de suppliques pour une année pontificale. K. SALONEN, op. cit., p. 26-28. L'étude ne prend pas en compte les suppliques de declaratoriis par lesquelles les suppliants sollicitent la déclaration de leur innocence à propos du crime dont ils sont accusés. Je remercie Mme K. Salonen pour les transcriptions qu'elle m'a fournies concernant les registres de Pie II. 8.Parmi ces 253 suppliques, 99 ont été recensées pour le pontificat de Pie II, ce qui représente $2,7 \%$ du nombre total de suppliques de diversis sous ce même pontificat. Pour 
un décompte des suppliques de la Pénitencerie sous Pie II, voir L. SchMUGge dir., Die Supplikenregister, op. cit., p. 57 et sq.

9.Lorsqu'un religieux commet un crime, sa correction peut se faire à un niveau local (couvent) ou régional (chapitres provinciaux ou généraux) lorsqu'il dépend d'un ordre. Le supérieur ou le chapitre général examine le cas, statue sur la culpabilité de la personne accusée et prononce une sentence. Voir notamment les sources des chapitres généraux des ordres de Cluny et Cîteaux, Statuts, chapitres généraux et visites de l'ordre de Cluny, G. C HARVIN éd., 9 vol., Paris, 1965-1982 (désormais abrégé en : CHARVIN) et Statuta Capitulorum Generalium Ordinis Cisterciensis, 1116-1786, J. CANIVEZ éd., 8 vol., Louvain, 1933-1941 (désormais abrégé en : CANIVEZ).

10.À cet égard, les précautions méthodologiques mises en exergue par C. Gauvard pour les lettres de rémission délivrées par la Chancellerie du roi de France à la fin du Moyen Âge peuvent s'appliquer aux suppliques de la Pénitencerie. Comme le montre W. Müller, ces deux types de documents émanent d'une autorité judiciaire suprême, qui les délivre à la requête d'un particulier. L'octroi de la grâce résulte d'un jeu complexe entre souci d'exactitude et atténuation du délit. C. GAUVARD, «De grace especial ». Crime, État et société en France à la fin du Moyen Âge, 2 vol., Paris, 1991, chapitre 2 ; W. MÜLLER, op. cit.

11.« Si quis suadente diabolo huius sacrilegii uicium incurrerit, quod in clericum uel monachum uiolentas manus iniecerit, anathematis uinculo subiaceat, et nullus episcoporum illum presumat absoluere, nisi mortis urgente periculo, donec apostolico conspectui presentetur, et eius mandatum suscipiat. » Décret de Gratien, C. 17.4.29, FRIEDBERG, I, col. 822. La réserve est le droit de juridiction dont le supérieur dans la hiérarchie ecclésiastique déclare conserver l'exercice exclusif. Sur le droit de réserve, voir A. Fossier, op. cit., p. 210 et sq.

12.Décrétale Si vero, Liber Extra, X 5.39.3, FRIEDBERG, t. II, col. 890 et X 5.39.1, FRIEDBERG, t. II, col. 889-890. A. LEFEBVRE-TEILLARD, « L'excommunication dans le droit canonique classique (XII ${ }^{\mathrm{e}}$-XIII ${ }^{\mathrm{e}}$ siècle) », dans J. HOAREAU-DODINAU dir., La Peine. Discours, pratiques et représentations, Limoges, 2005, p. 31-44.

13.« Monachi vero et canonici regulares, quocumque modo se in claustro percusserint, non sunt ad apostolicam sedem mittendi, sed secundum providentiam et discretionem sui abbatis disciplinae subdantur ", X 5.39.2, FRIEDBERG, t. II, col. 890.

14.« De monialibus tua a nobis fraternitas requisivit, per quem eis sit beneficium absolutionis impendendum, si vel in se invicem vel conversos vel conversas suas, aut clericos etiam, in suis monasteriis servientes, manus iniecerint temere violentas. Super hoc igitur tuae consultationi taliter respondemus, ut auctoritate nostra per episcopum, in cuius dioecesi earum monasteria fuerint, absolvantur », X 5.39.33, FRIEDBERG, t. II, col. 903. 15.« Canonica constitutione cavetur quod monachi et canonici regulares, quocumque modo se in claustro percusserint, non sunt ad apostolicam sedem mittendi, sed secundum discretionem et providentiam sui abbatis disciplinae subdantur. Quodsi abbatis discretio ad eorum correctionem non sufficiat providentia est dioecesani episcopi adhibenda, nisi excessus difficilis fuerit et enormis, propter quem merito ad ecclesiam Romanam sit recursus habendus ", X 5.39.50, FRIEDBERG, t. II, col. 910.

16.J. THÉRY, « Enormia. Éléments pour une histoire de la catégorie de « crime énorme » au second Moyen-Âge ", Annuaire EHESS, Comptes-rendus des cours et conférences 2005-2006, Paris, 2007, p. 535-537.

17.« [...] Si qui cum ad conversionem eius accedunt vel postea professi fuerunt qui per appositione ignis aut pro violenta manuum iniectione in clericum vel aliam religiosam 
personam vinculo teneantur excommunicationis astricti vel qui excommunicati communicaverint de auctoritate nostra facultatem habeat absolvendi et penitentia iniungendi quam videritis salutarem.» Alexandre III, Cum sitis, 7 mars (pas d'indication d'année), Privilegia ordinis Cisterciensis, J. de CIREY éd., 1491, fo 8 r. Ce privilège est confirmé en 1216 par Honorius III, CANIVEZ, I, 1216, 19. Le chapitre général de 1220 distingue cependant l'absolution du crime et la dispense de l'irrégularité contractée à cette occasion. Il précise que les abbés ne peuvent dispenser les moines de l'irrégularité contractée pour les cas d'homicides et de violence à l'encontre d'un évêque, d'un abbé, d'un clerc séculier et pour les cas de mutilation et d'« effusion de sang énorme ». Ces cas reviennent à la compétence du chapitre général. CANIVEZ, I, 1220, 29. Concernant la violence interne au cloître et la législation cistercienne, voir T. FÜSER, Mönche im Konflikt, Zum Spannungsfeld vom Norm, Devianz und Sanktion bei den Cisterziensern und Cluniazensern (12, bis frühes 14 Jahrhundert), Münster, 2000, p. 123-131.

18.Grégoire IX, Cum monachos, 23 janvier 1234, J. de CIREY, op. cit., fo $15 \mathrm{v}$. Le pape confère cette compétence aux abbés mais aussi aux prieurs. Alexandre IV étend, par la suite, cette compétence à tout prêtre à qui l'abbé local aura délégué sa juridiction, Cum sicut ex parte, $1^{\text {er }}$ février 1255, J. de CIREY, op. cit., fo $20 \mathrm{v}$.

19.« Qui in monachum vel conversum manus violentas iniecerit, quia ipso facto excommunicatus est, cautius evitetur ab omnibus, donec ab abbate vel priore, si abbas in absentia sua ipsi licentiam absolvendi dederit, absolveatur. Qui autem in abbatem simili modo manus iniecerint, sententie conspiratorum per omnia subiacerunt, et a Summo Pontifice sunt absolvendi, Les Codifications cisterciennes de 1237 et de 1257», B. LUCET éd., Paris, 1977, C VI 11 et D VI 11, p. 277. La codification de 1237 est la troisième codification de l'ordre de Cîteaux. Des collections systématiques de statuta avaient déjà été produites en 1202 et 1220 . Ces « livres de définitions » opèrent une sélection dans les décisions prises par les chapitres généraux précédents. Ils sont rédigés par les définiteurs, qui reçoivent leur autorité du chapitre général et ont des compétences très larges en matière législative et disciplinaire. L'ordre de Cluny comprend également des définiteurs.

20. [...] Ut singuli vestrum in conventibus sibi commissis, praedictis fratribus constitutis ibidem absolutione ac dispensatione indigentibus, sive priusquam Ordinem intraverint, sive postea in casibus excesserint memoratis, de consilio discretorum fratrum vestrorum qui litterati sunt et Deum timentes, impertiri valeatis absolutionis beneficium, et dispensare cum eis vobis autoritate praesentium concedimus facultatem, nisi adeo fuerit gravis et enormis excessus, quod merito sint ad Sedem Apostolicam destinandi ", Privilège d'Alexandre IV, Licet ad hoc, $1^{\mathrm{er}}$ octobre 1255 ?, dans L. MescheT, Privilèges de l'ordre de Cîteaux, recueillis et compilés de l'autorité du chapitre général, Paris, 1713, p. 43 et Regesta Pontificum Romanorum, A. POTTHAST éd., Berlin, 1873-1875, n¹6033. Le pape ne précise pas la nature des excès qui requièrent l'absolution pontificale.

21.« [...] Ut abbates cum suis subditis in illis casibus, in quibus excommunicatio seu irregularitas contrahitur, absolvere et dispensare valeat, dummodo tales non sint, ut propter ipsorum enormitatem merito sit ad Sedem apostolicam recurrendum », CANIVEZ, II, $1255, \mathrm{n}^{\circ} 1$.

22." [...] Ut subditos suos in casibus in quibus excommunicationis sententiam et notam irregularitatis incurrerint, absolvere et dispensare valeant cum eisdem, nisi a deo gravis fuerit et enormis excessus, quod merito sint ad sedem apostolicam destinandi. Placet et concedit capitulum generale ut beneficio et gratia supradicta sibi concessa, precedente discretione et maturo consilio utantur. Sed quia difficile est ad plenum scire casus seu 
excessus pro quibus sit ad sedem apostolicam vel etiam ad capitulum generale recurrendum, districte precipitur eisdem abbatibus ut casus in quibus absolverint vel dispensaverint et causas sue absolutionis et dispensationis, dispensatoribus ordinis, si tamen dicti casus dubitabiles fuerint, in sequenti capituli generali studeant fideliter intimare, ut per ipsum capitulum, si forte in aliquo erratum fuerit, ad correctionis et rectitudinis lineam reducatur », B. LUCET, op. cit., D IV 50, p. 252.

23.C. BосK, Les Codifications du droit cistercien, Westmalle, 1955 ; B. LUCET, « L'ère des grandes codifications cisterciennes (1202-1350) », Études d'histoire du droit canonique dédiées $\grave{a}$ G. Le Bras, Paris, 1965, t. 1, p. 249-262.

24.CHARVIN, III, p. 497.

25.Cf. note $\mathrm{n}^{\circ} 15$.

26.CHARVIN V, p. 133.

27. CANIVEZ, $\mathrm{V}, 1467, \mathrm{n}^{\circ} 22$.

28.Ibid., IV, $1440, \mathrm{n}^{\circ} 10$.

29.Ibid., IV, 1441, nº30.

30.Il faut attendre le XVI ${ }^{\mathrm{e}}$ siècle pour que l'homicide devienne un cas réservé, W. MÜLLER, op. cit., p. 776.

31.Asv, Penitenzieria Ap., Reg. Matrim. et Div. 14, fo 184r. Le moine demande l'absolution pour ses excès et la dispense pour son irrégularité.

32."Quod processit ad sanguinis effusionem licet modicam ", Asv, Penitenzieria Ap., Reg.

Matrim. et Div. 7, fo 206v-207r. Je remercie Mme Salonem pour la transcription.

33.Asv, Penitenzieria Ap., Reg. Matrim. et Div. 13, fo $121 v-122 r$.

34.Cette stratégie est d'autant plus nécessaire que le droit canonique multiplie les subtilités pour évaluer au plus près l'imputabilité d'un crime. S. KUTTNER, Kanonistische Schuldlehre von Gratian bis auf die Dekretalen Gregors IX. Systematisch auf Grund der handschriftlichen Quellen dargestellt, Vatican, 1935, réimpr. 1961 ; L. KÉRY, « Non enim homines de occultis, sed de manifestis iudicant. La culpabilité dans le droit pénal de l'Église à l'époque classique », Revue de Droit Canonique, 53, 2003, p. 311-336.

35.Asv, Penitenzieria Ap., Reg. Matrim. et Div. 3, fo 89 r, RPG II, 133, p. 17. En montrant que la blessure n'est pas mortelle, le suppliant prouve que l'homicide est involontaire. X 5.12.28, FRIEDBERG, t. II, col. 800.

36." Oculos sibi eruissent, deinde per quamdam viam oculorum cerebrum terebrassent, et alia pessima et horrenda his similia proferendo nulla prorsus dissimulationem admittentia ", CANIVEZ, V, 1461, nº46.

37.Le chapitre envoie cinq abbés. À part l'abbé de Cherlieu, fille de Clairvaux (diocèse de Besançon), les commissaires sont des abbés voisins de l'abbaye de Tironneau : La Trappe, Bonport (diocèse d'Evreux), la Cour-Dieu (diocèse d'Orléans), Vaux-de-Cernay (diocèse de Paris). Sur les commissions envoyées par le chapitre général, J. SAYERS, « English cistercian cases and their delegation in the first half of the thirteenth century ", Analecta Sacri Ordinis Cisterciensis, 20, Rome, 1964, p. 85-102.

38.Le chapitre général donne aux commissaires l'injonction suivante. «Et si huiusmodi fratrem Lucam in his aut aliis similibus culpabilem aut convictum invenerint, secundum exigentiam, qualitatem et quantitatem causarum puniant et corrigant. » CANIVEZ, V, 1461, $\mathrm{n}^{\circ} 46$.

39.Asv, Penitenzieria Ap., Reg. Matrim. et Div. 10, f॰ 117r, éd. F. TAMBURINI, op. cit., cas nº. 40.K. SALONEN, op. cit., p. 87-88. 
41.Comme pour les lettres de rémission accordées par le roi de France au Bas Moyen Âge, l'absolution pontificale est rendue possible par le refus de définir trop précisément le crime. C. GAUVARD, « Le jugement entre norme et pratique : le cas de la France du Nord à la fin du Moyen Âge ", dans G. JARITZ dir., Norm und Praxis im Alltag des Mittelalters und der frühen Neuzeit, Vienne, 1997, p. 27-38.

42.Asv, Penitenzieria Ap., Reg. Matrim. et Div. 12, fo $64 \mathrm{v}$. Le suppliant demande à être absous et dispensé.

43. Concernant le choix fait par les suppliants entre la Chancellerie et la Pénitencerie, $P$. ZUTSHI, «Inextricabilis curie labyrinthus. The Presentation of Petitions to the Pope in the Chancery and the Penitentiary during the Fourteenth and First Half of the Fifteenth Century ", dans A. MEYER, op. cit., p. 393-410.

44.CANIVEZ, V, 1472, n40. D’après une décrétale de Clément III, les légats peuvent absoudre les sentences d'excommunication pour violence envers une personne religieuse, X 5.39.20, FRIEDBERG, t. II, col. 896, R. C. FIGUeIRA, « Papal Reserved Powers and the Limitations on Legatine Authority ", dans J. R. SWEENEY et S. CHODORAW dir., Popes, Teachers, and Canon Law in the Middle Ages, 1989, p. 191-211; P. ostineLLI, «L'offerta della grazia. Dispense e assoluzioni concesse dai vescovi e inviati pontifici in Lombardia nel XV secolo ", dans A. MEYER, op. cit., p. 530-549.

45.Les taxes pour les lettres de la Pénitencerie sont toutefois moins élevées que dans les autres offices pontificaux, K. SALONEN, op. cit., p. 84 et W. MÜLLER, « The Price of Papal Pardon, New Fifteenth-Century Evidence », dans A. MEYER, op. cit., p. 457-481.

46.Ce chiffre englobe les chanoines de Prémontré et les chanoines dits de « l'Ordre de Saint Augustin ». L'expression désigne les chanoines réguliers vivant sous la règle de saint Augustin au sein de monastères indépendants, à qui une ébauche d'ordre est imposée par la papauté dans le cadre de la province ecclésiastique. G. LE BRAS et J. GAUDEMET, Histoire du droit et des institutions de l'Église en Occident, t. X, Paris, 1971, p. 529.

47.Les archives mentionnent un ordo sancti Benedicti. La formule ne fait pas référence à un ordre bénédictin structurel mais à un mode de vie religieuse s'inspirant de la règle de saint Benoît. Seuls certains monastères bénédictins s'organisent en congrégation et forment un ordre religieux.

48.Sur le chapitre général F. CYGLER, Das Generalkapitel im hohen Mittelalter. Cisterzienser, Praëmonstratenser, Kartäuser und Cluniazenser, Münster, 2002. 49.Au XIVe siècle, Benoît XII (1334-1342) tente d'uniformiser l'organisation des différents ordres par la bulle Summi Magistri (20 juin 1336) pour les Bénédictins et la bulle Ad decorem (15 mai 1339) pour les chanoines réguliers indépendants. Bullarium romanum, C. C oCQUELINES éd., Rome, 1741, t. 3, p. 214-240 et Chapters of the Augustinian Canon, H. E. SALTER éd., Oxford, 1922, p. 214-267.

50.CANIVEZ, V, 1459, n²0, nº $21 ; 1460, \mathrm{n}^{\circ} 120 ; 1460$, p. $72 ; 1461, \mathrm{n}^{\circ} 46 ; 1461, \mathrm{n}^{\circ} 119 ; 1462, \mathrm{n}$ ${ }^{\circ} 98 ; 1466, \mathrm{n}^{\circ} 13, \mathrm{n}^{\circ} 106 ; 1467, \mathrm{n}^{\circ} 22 ; 1469, \mathrm{n}^{\circ} 70, \mathrm{n}^{\circ} 109 ; 1472, \mathrm{n}^{\circ} 23, \mathrm{n}^{\circ} 27, \mathrm{n}^{\circ} 40$.

51.Asv, Penitenzieria Ap., Reg. Matrim. et Div. 20, fo 222r, RPG VI, 2151, p. 272.

52. CANIVEZ, IV, $1442, \mathrm{n}^{\circ} 34$.

53.« Olim postquam fuerat de quibusdam censuris et delictis [...] coram certo abbate visitatore seu reformatore et superiore dicti monasterii per nonnullos eius emulos accusata ", Asv, Penitenzieria Ap., Reg. Matrim. et Div. 23, fo 127r, RPG, VI, 2490, p. 315. La moniale demande à être absoute de ses excès (qui ne sont jamais précisés dans la supplique), du crime d'apostasie et à être transférée dans un autre ordre. 
54.Asv, Penitenzieria Ap., Reg. Matrim. et Div. 5, fo 203r, RPG, III, 225, p. 33. Le moine ne demande pas de dispense.

55.Asv, Penitenzieria Ap., Reg. Matrim. et Div. 9, fo 119vs, RPG, IV, 1291, p. 84.

56.L. Mayali montre comment, à la fin du Moyen Âge, la fuite hors du monastère est considérée comme un comportement dangereux et criminel, L. MAYALI, « Du vagabondage à l'apostasie. Le moine fugitif dans la société médiévale ", dans D. SIMON dir., Religiöse Devianz, Untersuchung zu socialen, rechtlichen und theologischen Reaktionen auf religiöse Abweichung im westlichen und östlichen Mittelalter, Francfort, 1990, p. 121-142.

57.En 1335, Benoît XII rappelle que la sanction doit être thérapeutique, Bullarium romanum , III, 2, 201-203.

58.Asv, Penitenzieria Ap., Reg. Matrim. et Div. 18, fo 178v. En revanche, il ne demande pas à être dispensé.

59. Concernant « ceux qui, souvent repris, ne veulent pas s'amender », la Règle de saint Benoît prescrit que « l'abbé prendra le couteau pour amputer, comme dit l'Apôtre : « Retranchez le pervers du milieu de vous » [...] de peur qu'une brebis malade ne contamine tout le troupeau ». La Règle de saint Benoît, A. de VoGÜE éd., Paris, 1972, XXVIII, 6-8. Selon Gratien, les clercs incorrigibles doivent être séparés de l'Église et ne peuvent bénéficier du pardon, Décret, C.24.3.14 et C.24.2.5, FRIEDBERG, I, col. 994 et 986. Outre la réitération de l'acte criminel, l'incorrigibilité suppose que le religieux ait déjà été corrigé et persévère dans la désobéissance. La supplique ne mentionne cependant pas de monition et de correction de la part du supérieur. Sur l'incorrigibilité, C. GAUVARD, "Justification and Theory of the Death Penalty at the Parlement of Paris in Late Middle Ages ", dans C. Allmand dir., War, Government and Power in the Late Medieval France, Liverpool, 2000. Sur l'expulsion, A. PFALLER, The ipso facto effected dismissal of religious, Washington, 1948.

60.Asv, Penitenzieria Ap., Reg.. Matrim. et Div. 5, fo 161v, RPG, III, 185, p. 27. Les raisons de l'apostasie ne sont pas spécifiées dans la supplique.

61. CANIVEZ, II, $1278, \mathrm{n}^{\circ} 14$.

62.Article rédigé dans le cadre d'un doctorat en cours portant sur la criminalité dans les communautés conventuelles en Occident médiéval ( $\mathrm{XII}^{\mathrm{e}}-\mathrm{XV}^{\mathrm{e}}$ siècle), sous la direction de $\mathrm{M}$. F. Collard. L'étude prend en compte le monde régulier dans son ensemble (moines, chanoines réguliers et frères mendiants) en raison de l'unification du mode de vie des réguliers à la fin du Moyen Âge. Je remercie M. W. Müller et Mme K. Salonen pour leur relecture et leurs conseils.

\section{RÉSUMÉS}

Les registres de la Pénitencerie apostolique de la seconde moitié $\mathrm{du} \mathrm{xv}^{\mathrm{e}}$ siècle contiennent des suppliques rédigées par des religieux (moines, chanoines réguliers et frères mendiants ou moniales), impliqués dans des actes de violence à l'encontre d'un confrère ou d'un clerc. Ces clercs réguliers demandent l'absolution du crime commis, de l'excommunication encourue ipso facto et une dispense pour l'irrégularité contractée. L'étude de la législation canonique en 
matière de violence exercée par des religieux à l'encontre d'une personne ecclésiastique montre cependant que les clercs réguliers ne sont pas astreints à recourir à Rome. La papauté a, en effet, progressivement délégué ce pouvoir aux prélats réguliers et séculiers, tout en gardant un droit de réserve sur les excès difficiles et énormes. De nombreux religieux recourent pourtant à l'office pontifical pour des cas de violence assez ordinaires, tels que des coups échangés entre confrères, qui pourraient être absous par leur supérieur, leur évêque ou un légat pontifical. Il s'agit généralement de religieux désobéissants et souvent apostats, qui tentent d'échapper à la correction de leurs supérieurs en sollicitant le pardon pontifical. Les lettres d'absolution accordées par la Pénitencerie constituent en effet des pièces décisives susceptibles d'influer sur les décisions des juridictions locales qui, si elles sont compétentes en la matière, se montrent peu clémentes avec les religieux délinquants.

Clerics Seeking Pardon: Petitions Brought before the Apostolic Penitentiary by Violent Regular Clerics in the Fifteenth Century. The registers of the Apostolic Penitentiary for the second half of the fifteenth century provide petitions from regular clerics (monks, canons, friars or nuns) who committed an act of violence (fight, assault, homicide) against another ecclesiastic. They asked for absolution for their crime and their ipso facto excommunication, and for dispensation from sacramental impediments. According to canon law, religious people who acted violently towards another ecclesiastic did not have to petition before the Penitentiary : abbots, priors, bishops and legates had indeed received power to grant absolution in these matters, the papal authority only reserving its power to absolve difficilis et enormis excesses. Nevertheless, many regular clerics kept submitting petitions for grace to the Penitentiary, despite the fact that it could be obtained locally, for instance in ordinary cases of violence such as fights between brothers or sisters. Such petitioners were often disobedient and apostate clerics, who tried to escape an abbot's punishment by seeking papal forgiveness. The letters granted by the Penitentiary carried enough power to pressure reluctant local jurisdictions to reintegrate the criminal clerics into their community.

\section{INDEX}

Mots-clés : absolution, communautés, excommunication, pénitencerie apostolique, religieuses, violence

Keywords : apostolic penitentiary, religious orders

\section{AUTEUR}

\section{ELISABETH LUSSET}

Université de Reims Champagne Ardenne, EA 2616, CERHIC, Maison de la Recherche, 57, rue Pierre Taittinger - F-51096 Reims Cedex. 